\title{
Nuclear hamiltonians: The question of their spectral predictive power and the associated inverse problem
}

\author{
J DUDEK ${ }^{1}$, B SZPAK $^{2}$, M-G PORQUET ${ }^{3}$, H MOLIQUE $^{1}$, \\ $\mathrm{K}_{\text {RYBAK }}{ }^{1}$ and B FORNAL ${ }^{2}$ \\ ${ }^{1}$ Institut Pluridisciplinaire Hubert Curien \\ Département de Recherches Subatomiques UMR7178, IN2P3-CNRS and \\ Université de Strasbourg, 23 rue du Loess B.P. 28, \\ F-67037 Strasbourg Cedex 2, France \\ ${ }^{2}$ The Niewodniczański Institute of Nuclear Physics, Polish Academy of Sciences, \\ ul. Radzikowskiego 152, Pl-31-342 Kraków, Poland \\ ${ }^{3}$ Centre de Spectrométrie Nucléaire et de Spectrométrie de Masse, \\ IN2P3-CNRS and Université Paris-Sud, Bâtiments 104 et 108, \\ F-91405 Orsay Campus, France \\ E-mail: Jerzy.Dudek@IReS.in2p3.fr, Bartlomiej.Szpak@ifj.edu.pl, \\ Marie-Genevieve.Porquet@csnsm.in2p3.fr, Herve.Molique@IReS.in2p3.fr, \\ Karolina.Rybak@IReS.in2p3.fr, Bogdan.Fornal@ifj.edu.pl
}

\begin{abstract}
.
In this article we introduce a subjective notion of the predictive power of nuclear Hamiltonians (an objective one does not exist) and examine it in the particular context of the single-nucleon energy spectra. We consider various types of uncertainties originating both from the experiment and theory stressing the dominating character of the theoretical errors. The latter originate from the complexity of the nuclear many body systems that is not matched adequately by the formalism behind the present day nuclear Hamiltonians. The related inverse problem is formulated and the presence of errors (ignorance, lack of knowledge) is parametrized in terms of the associated probability distributions. Various hypotheses concerning the input uncertainties ('numerical noise') are formulated and the impact of the input-uncertainties in the adjustment procedures down to the final parameter values and theoretical spectra is illustrated and discussed. A number of Open Problems are formulated and listed at the end of the article.
\end{abstract}

PACS numbers: 21.10.-k,21.10.Pc,21.60.-n,21.60.Jz,02.30.Zz,02.60.Ed 


\section{Introduction: Predictive power and the inverse problem}

In this article we formulate and discuss the problem of predictive power of nuclear theories focusing on the underlying properties of nuclear structure Hamiltonians. The notion of predictive power associated with a given theory, similarly to the very notion of probability, is subjectiveł. According to the common sense, the adjective 'predictive' signifies theory's capacity of providing realistic i.e. experiment-comparable outcome prior to the effectively performing the measurement. Again, the qualifying terms such as 'realistic' and 'comparable with experiment' are left to physicist's judgement.

We arbitrarily distinguish between two regimes of theory's functioning and the two related forms of predictive power called extraneous, from the Latin 'extraneus' (outside) and intraneous, from the Latin 'intraneus' (inside). The former adjective refers to the capacity of predicting the experimental results outside of the realm of the experimental input used to determine parametrisation of the theory, e.g. predictions for the super-heavy or exotic nuclei based on the parameter adjustments to the properties of, say, $\beta$-stable nuclei. Alternatively, the latter adjective refers to the capacity of predicting the properties of nucleus ' 2 ' using the parameter adjustments to the 'neighbouring' nuclei ' 1 ' and ' 3 '.

The very word 'prediction' refers to our lack of knowledge: We do not know what the binding energy of the nucleus ${ }_{126}^{322} \mathrm{Xx}_{196}$ is before we actually succeed in measuring it and thus in the best case we may try to 'predict it' before experimenting. However, we may try to quantify our lack of knowledge. Based on our experience with hundreds of other binding energies we may be able to estimate the probability within which, we believe, our theoretical prediction should apply. This does not offer any guarantee (as it is always the case when working within the realm of the probability calculus) - but diminishes our ignorance and possibly strengthens our (subjective) confidence§. In what follows we will illustrate these issues using realistic (phenomenological) mean field Hamiltonians and realistic experimental data.

The science of obtaining statistically significant result 'for the whole' out of the 'limited data-samples' is a sub-field of applied mathematics called inverse problem: the latter is usually formulated as follows. Given the experimental results called data $\{d\}$ and a set of parameters $\{p\}$ that must be determined by these data. Next, let all the operations leading within the considered theory from the set of parameters to the theoretical prediction of the data be abbreviated by $\hat{\mathcal{O}}$ : we write symbolically $\{d\}=\hat{\mathcal{O}}\{p\}$. In what follows, the data will represent the energies generated by a certain quantum Hamiltonian while $\{p\}$ - the set of its parameters. Calculating the energies out of the known parameter set is referred to as solving the direct problem, whereas determining the parameters out of predefined (usually experimental) energies - the related inverse problem. Since the need for the inverse problem solutions is

$\ddagger$ Indeed, the word probability does not have any unique/consistent definition, there exist two most frequently involved approaches dealing with probability, the 'frequentist' and the 'Bayesian' ones, not to forget a couple of others (Jeffrey's the so-called logical and Fischer's fiducial), together with over a dozen probability interpretations. In short: by entering the territory of probability distributions we introduce inevitably subjective criteria; in this text we follow the frequentist notion of probability.

$\S$ In their Introduction to Bayesian Scientific Computing, Calvetti and Somersalo [1] use the following dialogue to illustrate the similar context. [Origin: Umberto Ecco's 'The Name of the Rose'].

"So you don't have a unique answer to your questions?"

"Adson, if I had, I would teach theology in Paris."

"Do they always have a right answer in Paris?"

"Never", said William, "but there they are quite confident of their errors". 
overwhelmingly present in practically all the modern sciences ranging from geology, sociology, politics and medicine to physics and mathematics, there exists rich literature related to the underlying mathematical tools. The use of them was relatively seldom practiced in low-energy sub-atomic physics but we believe that our field of research has numerous possibilities to profit from this evolution.

Our analysis aims at Hamiltonians of contemporary microscopic theories such as Density Functional Theories including various realisations of the Nuclear Mean-Field Methods, Nuclear Shell-Model and Nuclear Phenomenological Model Hamiltonians. Despite an apparent diversity all these complex nuclear theories depend on a common factor: their performance and especially their predictive power may considerably profit from the modern advanced mathematical theories of statistically significant parameter determination. The performance of realistic theories depends crucially on the adequate selection of theory's adjustable parameters. The latter depends on the choice and the quality of the experimental-data input, and, what is strongly related, on the statistical significance $\|$ of the resulting parameterisation.

The parameter determination is strongly influenced by theoretical modelling since extraction of the experimental information often depends on model dependent procedures (examples will be given below). Moreover, theories themselves attempt a description of only a part of the physical reality whereas both the already established and yet undiscovered mechanisms have their impact on the experimental result. In this article we pose the problem of quantifying the unknown through probability distributions within the framework of what could be dubbed elements of theory of theoretical errors. Presenting our selection of Open Problems, we hope to contribute to intensifying the interest in this field and hopefully accelerating the associated research.

We have chosen to limit our discussion to the spectral properties of the nuclear systems i.e. the energy eigenvalues of the underlying Hamiltonians; other observables can be included in the discussion with no major difficulty. The illustrations (but not the considerations) are purposely limited to the problem of reproducing the singlenucleonic energies in spherical doubly-magic nuclei. This will allow us to illustrate examples of problems encountered when extracting the experimental information before injecting it into the parameter determination algorithms. The discussion of the parameter determination techniques is greatly facilitated by using a realistic context, but simple enough to be able to construct exactly soluble test-cases with the rapid solution possibilities. For that purpose we will select a toy-model Hamiltonian containing the spherical Woods-Saxon central potential together with the spin-orbit mean-field components. The reader is warned not to be mislead by the simplicity of the chosen toy model as opposed to complexity of the microscopic theories aimed at. Since the functioning of the inverse problem techniques is to far an extent not dependent on their practical realisations, we will try to profit from the presented examples to pose important, fundamental questions for the research to come.

\| The issue of statistical significance is not void of interdisciplinary interest as the authors of [2] observe with sarcasm: "Unfortunately, many practitioners of the parameter estimation never proceed beyond determining the numerical values of the parameter fit. They deem a fit acceptable if a graph of data and model 'look good'. This approach is known as chi-by-the-eye. Luckily, its practitioners get what they deserve". Expressed more explicitly: parametrisations obtained in this way risk to be unstable, statistically worthless and thus useless for extrapolations of any kind and in particular killing the chances for an extraneous predictive power. 


\section{Experimental information, built-in modelling and related uncertainties}

The following discussion shows clearly, that the so-called experimental single particle energies in spherical nuclei are highly complex, model dependent objects. The possibly precise extracting of the energy information from experiment requires well designed instrumentation, involves weak signals whose analysis deserve increased statistics data - conditions that are not easy to meet especially for the exotic nuclei such as a very important ${ }^{132} \mathrm{Sn}$ nucleus. Its importance consists in the fact that together with ${ }^{208} \mathrm{~Pb}$ these two spherical doubly-magic nuclei form the last strongholds when exploring the exotic heavy and/or super-heavy nuclei.

The experimental information about the single-particle level-structure of eveneven doubly-magic nuclei with the proton number $Z_{0}$ and neutron number $N_{0}$ originates mainly from the $\left(Z_{0} \pm 1\right)$ and $\left(N_{0} \pm 1\right)$ nuclear neighbours. Population of the single-particle or single-hole nucleonic levels in the $\left(A_{o} \pm 1\right)$ residual nuclei is favoured through the light-projectile direct nucleon-transfer reactions such as $(\mathrm{d}, \mathrm{p})$, $\left({ }^{3} \mathrm{He}, \mathrm{d}\right),(\mathrm{t}, \mathrm{d})$ or $(\mathrm{p}, \mathrm{d}),\left(\mathrm{d},{ }^{3} \mathrm{He}\right),(\mathrm{d}, \mathrm{t})$ on mass- $A_{0}$ doubly-magic nuclei. In principle, the results of such experiments directly reveal the $j$-shell structure around the magic energy gaps, since each measured level does correspond to one single-particle or singlehole energy state and the reactions of this type are capable of providing the excitation energy, angular momentum and parity of the states in question. It is well known that all the $j^{\pi}$ characteristics of the low-lying states measured in the $\left(A_{o} \pm 1\right)$ residual nuclei close to the stability line are in agreement with the expected sequence of singleparticle energy-levels in a spherical mean field containing the spin-orbit potential. Nevertheless the separation energies of these low-lying states are not necessarily the single-particle energies we are looking for. There are often other levels with the same $j^{\pi}$ in the same energy region, arising from excited core configurations or from coupling to the collective vibration of the surface, which mix (i.e. strongly couple among themselves through nuclear interactions thus giving rise to big matrix elements) with the unperturbed single-particle state. As a result, even though the single-nucleon strength may be maximum for the first state with the given $j^{\pi}$, it is distributed among several other nuclear levels as well. To recover the single-nucleon energy, $\varepsilon_{\kappa}$, one computes the centre of gravity of the $j^{\pi}$ group using weight factors proportional to the excitation strengths observed in the transfer reaction, viz.:

$$
\varepsilon_{\kappa}=\sum_{k} S(\kappa, k) e_{k}^{e x c} \text { with the sum rule } \sum_{k} S(\kappa, k)=1 .
$$

Above, $e_{k}^{e x c}$ is the measured energy of the $k^{\text {th }}$ level, and $S(\kappa, k)$ is its spectroscopic factor, i.e. the probability that the wave-function of the measured level $k$ contains the single particle state $\kappa$. This procedure has been justified by M. Baranger [3], from a theoretical point of view. One has to stress that the appropriate use of (1) to calculate the single-particle energy $\varepsilon_{\kappa}$ requires that the sum rule is fulfilled i.e. that (i) All the $k$-levels to which the single-particle state $\kappa$ contributes are identified, and: (ii) Spectroscopic factors have been determined precisely i.e. without any major bias.

The weighted-average procedure may be modified through a normalisation

$$
\varepsilon_{\kappa}=\left[\sum_{k} S(\kappa, k) \cdot e_{k}^{e x c}\right] /\left[\sum_{k} S(\kappa, k)\right]
$$

with the hope that even if, in view of the large experimental errors on the spectroscopic factors, the sum rule $\sum_{k} S(\kappa, k)=1$ is poorly approximated, the impact of the implied uncertainties is diminished in this way. 


\subsection{Extracting single-nucleon energies and associated uncertainties}

A typical experiment on one-nucleon transfer reaction provides the spectrum of the light ejectiles, i.e. the number of counts as a function of the ejectile energy, for many detection angles in the laboratory system. These raw data are analyzed in order to deduce, for each state populated in the residual nucleus, the differential cross section, $\frac{d \sigma}{d \Omega}$, as a function of $\theta_{c m}$, the ejectile emission angle in the centre-of-mass system. Depending on the experimental conditions, the functions $\frac{d \sigma}{d \Omega}\left(E_{k}\right)=f\left(\theta_{c m}\right)$ are not always determined with enough precision. For instance the detection efficiency may be too small to measure some weakly populated states, the knowledge of detection efficiency may be too fragmentary to compute the absolute value of $\frac{d \sigma}{d \Omega}$, or the angular range of detection may be too narrow to obtain the full angular distribution.

The above measurements provide the information about the single-nucleon stateenergies. Firstly, the measured angular distribution determines the orbital angular momentum $l$ of the 'stripped' or 'picked-up' nucleon and this assigns the angular momentum and the parity to each level. The remaining ambiguity, whether the level under consideration corresponds to $j=(l+1 / 2)$ or $j=(l-1 / 2)$ configuration, can be removed when using polarized beams. Secondly, the absolute value of the cross section gives a measure of the proportion in which a particular single-particle state contributes to the given level $j^{\pi}$. More precisely, whereas the former aspect is well under control for standard one-nucleon transfer reactions, the extraction of the socalled 'experimental' value of the spectroscopic factor needs precise modelling of the reaction process, since it is obtained through dividing the experimental cross section by the theoretical value calculated under the assumption that the populated state is a pure single-particle/hole state,

$$
\left.\frac{d \sigma}{d \Omega}\left(\theta, E_{i}\right)\right|_{\text {exp }}=\left.S(\kappa, i) \cdot \frac{d \sigma}{d \Omega}\left(\theta, E_{\kappa}\right)\right|_{t h}
$$

From the above expression, the spectroscopic factor should be a constant independent of the angles, implying that the theoretical cross section would reproduce experiment throughout the entire angle range. In practice this is not the case and to minimize the error, the value of $\mathrm{S}$ is determined at the angle where the experimental cross section has its maximum. This is clearly one of the aspects contributing to the final uncertainties of the extracted level-energies.

The simplest useful reaction model, the Distorted Wave Born Approximation, assumes a direct, one-step transfer process in which the transfers to specific states are individually weak and may be treated using perturbation theory. The distorted waves are provided by optical model potentials that describe the appropriate elastic scattering in the entrance and exit channels. It is important to emphasize that various types of optical potentials, while fitting the elastic scattering data equally well, may give very different results for the shape of the angular distribution of the ejectile as well as the values of the spectroscopic factors of the populated states. Moreover, two potentials are needed, the one of which binds the transferred nucleon in the exit channel as well as the one binding it in the entrance channel. The geometric parameters of the binding potentials are rather poorly constrained so that they are subject to rather large parametric uncertainties. Since the theoretical cross section scales with the radius parameter, the derived spectroscopic factor can vary up to $30 \%$ depending of the chosen parameter values.

The fact that the fit to the measured angular dependence of the cross-section, $\left.\frac{d \sigma}{d \Omega}\left(\theta, E_{i}\right)\right|_{\text {exp }}$, is not satisfactory in the whole angular range signifies that in many 
cases the reaction model is too simple to describe the reality satisfactorily. One may expect that a part of the discrepancies originates form the nuclear structure effects not taken into account in the model as well as the influence of two-step transfer processes including inelastic excitation of the target, projectile or residual nucleus. Using more elaborate models for the transfer reactions such as the Coupled Channel Born Approximation or Coupled Reaction Channels produces better fits whereas the values of spectroscopic factors are often strongly altered.

It follows that the ambiguities related to the spectroscopic factors may be as large as $30 \%$ in absolute values as a result of otherwise careful analyses. Thus even though a level strongly populated in one-nucleon transfer reaction has been interpreted as a pure single-particle state (because the value of its spectroscopic factor has been found close to unity) such a result has still to be counter-checked by verifying whether other states with the same value of spin and parity are not populated as well using the same reaction. This is a very difficult task, dealing with large energy ranges and very low values of the cross sections making the identification of the states a very tough issue. In addition, it is worth recalling that the transfered angular momentum strongly depends on the projectile energy. Therefore in order to obtain a complete picture of all the states with all the expected $j^{\pi}$ values, one would ideally need to combine experimental results obtained in a large range of bombarding energies and using various projectiles. Unfortunately, such complete studies are seldom available in the literature and remain an Open Problem in this type of research.

\subsection{Important textbook case: Single-nucleon energies in ${ }^{208} \mathrm{~Pb}$}

The doubly-magic, spherical nucleus ${ }^{208} \mathrm{~Pb}$ is considered to be one of the best studied doubly-magic nuclei. It will be instructive to discuss the analysis of its single-particle structure especially in view of an attempt of extending the analysis results to the much poorer known nucleus of ${ }^{132} \mathrm{Sn}$, or even lighter doubly magic nuclei.

2.2.1. Excited states of the ${ }^{208} \mathrm{~Pb}$ core. The lowest excited state of the ${ }^{208} \mathrm{~Pb}$ nucleus, at $2.614 \mathrm{MeV}$, is the well-known collective-oscillation octupole state of $\mathrm{B}(\mathrm{E} 3)=40 \mathrm{~W} . u$. At higher excitation energies corresponding to about 3-to-4 MeV, there are several states interpreted mainly in terms of combinations of one-particle one-hole $(1 p-1 h)$ excitations, some of them having negative parity (such as the $5^{-}$level at $3.198 \mathrm{MeV}$ ) or positive parity (such as the $2^{+}$level at $4.085 \mathrm{MeV}$ ). The octupole vibration of ${ }^{208} \mathrm{~Pb}$ is the most important in the fragmentation of the single-particle strengths in the neighbouring odd-A nuclei because of both its low energy and its strong collectivity, nevertheless the other states may also contribute to the fragmentation.

2.2.2. Deconvolution of the single-nucleon states around gaps at $Z_{0}=82$ and $N_{0}=126$. The four odd-A nuclei close to ${ }^{208} \mathrm{~Pb}$ viz. ${ }_{83}^{209} \mathrm{Bi}_{126},{ }_{81}^{207} \mathrm{Tl}_{126},{ }_{82}^{209} \mathrm{~Pb}_{127}$ and ${ }_{82}^{207} \mathrm{~Pb}_{125}$, had been studied using several one-nucleon pick-up or stripping reactions, some thirty years ago. At that time, the experiments had only focused on the dominant singleparticle or single-hole states, lying in a restricted range of excitation energies. Except for a few cases, all the observed states were considered as pure single-nucleon states. Their spectroscopic factors were assumed to be close to unity and used to test the Distorted Wave Born Approximation procedures.

The resulting, lowest-lying excited states, denoted $\mathrm{e}_{1}^{e x c}$, are presented in table 1. 
Table 1. Proton and neutron levels around the two magic gaps at $Z_{0}=82$ and $N_{0}=126$. Symbol $e_{1}^{e x c}$ represents the energy of the lowest state of each spin value measured in ${ }_{83}^{209} \mathrm{Bi},{ }_{81}^{207} \mathrm{Tl},{ }^{209} \mathrm{~Pb}_{127}$, and ${ }^{207} \mathrm{~Pb}_{125}$. When written in bold, this state is usually considered as a pure single-nucleon state, i.e. with a spectroscopic factor close to unity [4]. The energies $\varepsilon_{\kappa}$ of the single-nucleon states are computed with (2), taking into account the $\mathrm{N}$ fragments which have been measured in the quoted references. The shift in energy is the difference between the energy $\mathrm{e}_{1}^{e x c}$ and the average energy, $\varepsilon_{\kappa}$. All the energies are given in $\mathrm{MeV}$.

\begin{tabular}{|c|c|c|c|c|c|c|c|c|c|}
\hline \multicolumn{5}{|c|}{ Proton levels around $Z=82$} & \multicolumn{5}{|c|}{ Neutron levels around $N=126$} \\
\hline Level & \multicolumn{4}{|c|}{ States in ${ }^{209} \mathrm{Bi}$} & Level & \multicolumn{4}{|c|}{ States in ${ }^{209} \mathrm{~Pb}$} \\
\hline & $\mathrm{e}_{1}^{e x c}$ & $\mathrm{~N}$ [ref] & $\varepsilon_{\kappa}$ & shift & & $\mathrm{e}_{1}^{e x c}$ & $\mathrm{~N}[\mathrm{ref}]$ & $\varepsilon_{\kappa}$ & shift \\
\hline$\pi h_{9 / 2}$ & 0.0000 & $1[5]$ & 0 & 0 & $\nu g_{9 / 2}$ & 0.0000 & 1 & 0 & 0 \\
\hline$\pi f_{7 / 2}$ & 0.8963 & $2[5]$ & 1.31 & 0.41 & $\nu i_{11 / 2}$ & 0.7788 & 1 & 0.779 & 0 \\
\hline$\pi i_{13 / 2}$ & 1.6086 & $5[5]$ & 1.97 & 0.36 & $\nu j_{15 / 2}$ & $1.423(1)$ & $4[8]$ & 1.77 & 0.35 \\
\hline$\pi f_{5 / 2}$ & 2.8262 & $5[5]$ & 3.44 & 0.61 & $\nu d_{5 / 2}$ & 1.5671 & - & - & - \\
\hline$\pi p_{3 / 2}$ & 3.1195 & - & - & - & $\nu s_{1 / 2}$ & 2.0322 & - & - & - \\
\hline \multirow[t]{2}{*}{$\pi p_{1 / 2}$} & $3.633(4)$ & - & - & - & $\nu g_{7 / 2}$ & $2.491(1)$ & - & - & - \\
\hline & & & & & $\nu d_{3 / 2}$ & $2.538(2)$ & - & - & - \\
\hline Level & \multicolumn{4}{|c|}{ States in ${ }^{207} \mathrm{Tl}$} & Level & \multicolumn{4}{|c|}{ States in ${ }^{207} \mathrm{~Pb}$} \\
\hline & $\mathrm{e}_{1}^{e x c}$ & $\mathrm{~N}$ [ref] & $\varepsilon_{\kappa}$ & shift & & $\mathrm{e}_{1}^{e x c}$ & $\mathrm{~N}$ [ref] & $\varepsilon_{\kappa}$ & shift \\
\hline$\pi s_{1 / 2}$ & 0.0000 & $3[9]$ & 0.10 & 0.10 & $\nu p_{1 / 2}$ & 0.0000 & 1 & 0 & 0 \\
\hline$\pi d_{3 / 2}$ & 0.3510 & $1 \quad[9]$ & 0.351 & 0 & $\nu f_{5 / 2}$ & 0.5697 & 1 & 0.570 & 0 \\
\hline$\pi h_{11 / 2}$ & 1.3481 & $2[9]$ & 1.44 & 0.10 & $\nu p_{3 / 2}$ & 0.8978 & 1 & 0.898 & 0 \\
\hline$\pi d_{5 / 2}$ & 1.6827 & $6 \quad[9]$ & 2.08 & 0.40 & $\nu i_{13 / 2}$ & 1.6334 & $9 \quad[6]$ & 2.4 & 0.8 \\
\hline$\pi g_{7 / 2}$ & $3.474(6)$ & $5[9]$ & 4.18 & 0.71 & $\nu f_{7 / 2}$ & 2.3399 & $4[7]$ & 3.0 & 0.7 \\
\hline & & & & & $\nu h_{9 / 2}$ & $3.414(2)$ & - & - & - \\
\hline
\end{tabular}

Some years later, by using other transfer reactions at higher bombarding energies, many cases of fragmentation have been observed. This allows us to calculate several values of $\varepsilon_{\kappa}$ using (2), as shown in table 1 . Unfortunately, these detailed results are not available for all the average energies associated with the whole set of single-nucleon levels of interest. The proton levels were studied in quite some detail, except for the highest-excited ones lying above $Z_{0}=82$. It turns out that because of their low orbital momentum, the population of the latter is not favoured in the proton-stripping reaction $\left({ }^{4} \mathrm{He}, \mathrm{t}\right)$ at $80 \mathrm{MeV}$ bombarding energy [5]. The fragmentation of the neutron levels is not very well documented; for instance, the fragments of the $\nu j_{15 / 2}$ level in ${ }^{209} \mathrm{~Pb}$ were sought because of the expected mixing with the $\nu g_{9 / 2} \otimes 3^{-}$coupling, whereas none of the high-lying-level fragments were precisely studied. Similarly, no fragment of the low-lying $\nu p_{3 / 2}$ level in ${ }^{207} \mathrm{~Pb}$ could be identified in the reactions $(\overrightarrow{d, t})$ at $200 \mathrm{MeV}$ bombarding energy [6] or $\left({ }^{3} \mathrm{He}, \alpha\right)$ at $70 \mathrm{MeV}$ [7], because of the strong mismatch in transfered angular momentum. Those, together with previously mentioned uncertainties prolongate the list of experimental Open Problems whose solutions may contribute to narrowing the error distributions as discussed below. 
2.2.3. Impact of collective excitations: particle-vibration coupling. The differences between $\mathrm{e}_{1}^{e x c}$ and $\varepsilon_{\kappa}$ amount to several hundreds of $\mathrm{keV}$ (see table 1). Such shifts can be partly attributed to the octupole coupling, as in each of the four odd-A nuclei, there are couples of single-particle levels with $\Delta l=3$ (and $\Delta j=3$ ):

$$
\left.\left.\begin{array}{ccc}
\pi f_{7 / 2} & \leftrightarrow & \pi i_{13 / 2} \\
\pi h_{11 / 2} & \leftrightarrow & \pi d_{5 / 2}
\end{array}\right\} \text { and } \begin{array}{ccc}
\nu g_{9 / 2} & \leftrightarrow & \nu j_{15 / 2} \\
\nu i_{13 / 2} & \leftrightarrow & \nu f_{7 / 2}
\end{array}\right\}
$$

Within the particle-vibration-coupling approach [10], we expect two fragments for each level [one of them related to the coupling between the single-particle lower- $l$ wave-function and the octupole $3^{-}$phonon wave-function coupled to the wave-function with the higher-l, say $\left.l^{\prime},\left(l \otimes 3^{-}\right)_{l^{\prime}}\right]$ and the implied shift in energy is expected to be a fraction of $2.614 \mathrm{MeV}$, the energy of the collective octupole state of the core (see figure 10 of [10]). Even though the actual number of fragments is usually greater than 2 , the mean value of the shifts $(0.4 \mathrm{MeV})$ might be linked to $2.614 \mathrm{MeV}$ what would give about $15 \%$ in terms of energy. Noteworthy is the fact that the shifts calculated in ${ }^{207} \mathrm{~Pb}$ are markedly larger, close to $30 \%$ of the $3^{-}$energy; but in that case the two levels of interest are very far from the Fermi level, lying in the energy range in which the mixing with many other configurations is expected.

Similarly, the quadrupole vibration can be at the origin of the fragmentation involving couples of single-nucleon states, characterized by $\Delta l=2$ (and $\Delta j=2$ ), such as $\pi h_{9 / 2} \leftrightarrow \pi f_{5 / 2}, \pi s_{1 / 2} \leftrightarrow \pi d_{5 / 2}$, and $\pi d_{3 / 2} \leftrightarrow \pi g_{7 / 2}$. Once more, we can rate the mean shift in energy (around $0.6 \mathrm{MeV}$, see table 1) in proportion of the $2^{+}$energy, what gives about $15 \%$, in terms of energy.

The above estimates and the information in table 1 have been used to model the typical error probability distributions, cf. Sects. 3-5. But we wish to emphasize, anticipating the conclusions from the results presented below, that the experimental information concerning not only the error estimates but also simply the too low a number of the experimental levels is a vital Open Problem in the determination of the nuclear Hamiltonians especially for lighter doubly-magic nuclei for which there are much fewer single-particle levels known at present (compared to ${ }^{208} \mathrm{~Pb}$ ).

\section{In-exact theories, random variables and error probability distributions}

In what follows, we arbitrarily oppose two, as it seems extreme types of modelling: a. Academic modelling designed to clarify functioning of certain physical mechanisms in abstraction from the detailed experimental data, as for instance over-viewing certain general features of multi-phonon oscillations, and: b. Realistic modelling, optimised to predict and/or to reproduce the experimental data related to real physical systems, as for instance the energy spectra of atomic nuclei. The first family of such theories is sometimes referred to as exact-, while the other one - due to a number of factors discussed in some detail below - may lead to what is referred to as in-exact theories.

Inexact theories pertain to the description of physical systems whose complexity exceeds our present-day theoretical knowledge. For instance, an insufficient knowledge of elementary interactions may not allow to built up a description of adequate manybody interactions. Alternatively, the complexity of physical systems may require that complications of the formalism go beyond the present-day level of human motivation (and capacities) - in particular if confronted with the lack of adequate contemporary mathematical tools or numerical capacities. Both types of discussed theories do not include all of the already discovered elements of knowledge and must not pretend to any 
completeness in describing the full reality. Therefore they must contain inadequacies leading us directly to the notion of the theoretical errors.

From the mathematical point of view theoretical and measurement errors are seen as random variables. It then follows that the observables which in the exact theories would have been represented just by real numbers - in the inexact theories turn into random variables (within certain intervals) and the associated probability distributions. This article focuses on the combined theory and experimental uncertainties and errors from the point of view of the associated probability distributions.

\subsection{Realistic in-exact theories and underlying postulates}

Realistic modelling and associated theories, aim at describing with the possibly highest fidelity a selected class of physical systems and phenomena. Such theories are not only based on certain experimental input but also aim at predicting and/or interpreting the outcome of measurements. We do not suggest that realistic theories are necessarily inexact. However, in the nuclear structure theory context this will be manifestly the case: in what follows the term realistic theory will be synonymous with inexact.

3.1.1. Realistic theories and the inverse problem. The number of experiments on any class of processes or systems is necessarily finite and, moreover, 'infinitesimally small' as compared to the number of all similar processes or systems that can be found in nature. Nevertheless, humans wish to model infinitely many physical situations using the experience with the very restricted sets of data below referred to as samples. This is what, among others, the inverse problem is about: using the results of observations to infer the values of the parameters characterizing the system under investigation. By doing so, equipped with a mathematical model, we may proceed to retrieve the information about the unknown quantities of interest by an indirect measurement. For instance by directly measuring the positions of say, $1 \mathrm{~s}_{1 / 2}$ and $1 \mathrm{~d}_{5 / 2}$ levels in a certain nucleus we may hope to be able to infer through modelling the energy of the $1 \mathrm{~d}_{3 / 2}$ level thus obtaining some information indirectly.

3.1.2. Realistic theories and errors of modelling. From now on we consider quantum Hamiltonian systems i.e. the physical systems whose states can be described in quantum mechanics with the help of the realistic energy operator

$$
\hat{H}=\hat{T}+\hat{V}(\hat{x}, p): \quad \hat{H} \psi_{n}=e_{n} \psi_{n} \rightarrow e_{n}=e_{n}(p) .
$$

Above, $\hat{x}$ represents observables (operators) such as position, momentum, spin, isospin etc. and $p$ - the full set of Hamiltonian parameters. Obviously the results of the theory, here the $n_{b}$ bound-state discrete energies $e_{n}$, for $n=1,2, \ldots n_{b}$, are known functions of parameters provided we have determined the parametric dependence of $\hat{H}$ on $p$ from the data. However, the data are known only within experimental uncertainties which may include in addition to the usual instrumental errors also the errors coming from the model-dependence in the data extraction-procedures as discussed in Sect. 2.

Moreover, working with inexact theories introduces inadequacies between the experimental data and the theory predictions. Indeed, all mechanisms present in the physical system, known or still unknown up to date, contribute to the measurement's outcome while only part of those mechanisms are modelled within the Hamiltonian and thus only this part contributes to the theoretical spectra. Let the experimental 
data be represented by the set of discrete energies $\left\{\varepsilon_{k}\right\}$ with $k=1,2, \ldots k_{\varepsilon}$; observe in passing that in general $k_{\varepsilon}<n_{b}$, more precisely, $k_{\varepsilon} \ll n_{b}$, but it may happen that $k_{\varepsilon}$ is comparable to $n_{p}$, the number of parameters: $p \leftrightarrow\{p\} \equiv\left\{p_{1}, p_{2} \ldots p_{n_{p}}\right\}$. By taking into account the uncertainties and using obvious notation we may write:

$$
\text { Theory } \rightarrow e_{n}=e_{n}^{t r u e}+\delta e_{n} \text { and } \varepsilon_{k}=\varepsilon_{k}^{t r u e}+\delta \varepsilon_{k} \leftarrow \text { Exp. }
$$

Above, $e_{n}^{\text {true }}$ and $\varepsilon_{k}^{\text {true }}$ are abstract 'exact' theory and 'exact' measurement results that are introduced only to facilitate the discussion of the principles but they do not appear explicitly it the final results. The theoretical and the experimental errors, denoted $\delta e_{n}$ and $\delta \varepsilon_{k}$, respectively, represent our lack of knowledge and thus, from the point of view of mathematics, they can be seen as continuous random variables. Undesired effects of the missing knowledge can be diminished through experience: for instance we may learn that a certain experimental outcome is more probable than the other. By introducing explicitly the implied probability distributions that quantify our lack of knowledge, we explicitly introduce subjective elements into considerations.

In this way we arrive at what is known in applied mathematics as statistical inference (or induction) problem and the idea of random sampling to infer certain unknown aspects of the studied system (statisticians would say: population).

The usual approach when determining Hamiltonian parameters from the set of data $\left\{\varepsilon_{k}\right\}$ is to minimize the generalized distance between the theory predictions and the data points. Such a distance can be, up to a normalisation, defined as

$$
\chi^{2}(p) \sim \sum_{k=1}^{k^{e x p}} w_{k}\{\underbrace{\left[\varepsilon_{k}^{\text {true }}+\delta \varepsilon_{k}\right]}_{\text {experiment }}-\underbrace{\left[e_{k}^{\text {true }}+\delta e_{k}\right]}_{\text {theory }}\}^{2} \rightarrow \frac{\partial \chi^{2}}{\partial p_{j}}=0,
$$

where $w_{k}$ are physicist's choice positive weight factors. Let us shorten the notation and write $\delta e_{n} \equiv \bar{e}_{n}$ and $\delta \varepsilon_{k} \equiv \bar{\varepsilon}_{k}$. Let the experimental and theoretical probability distributions associated with each of these variables be $\bar{\varepsilon}_{k} \leftrightarrow P_{k}^{e}\left(\bar{\varepsilon}_{k}\right)$ and $\bar{e}_{n} \leftrightarrow P_{n}^{t}\left(\bar{e}_{n}\right)$. In (7) there appear sums (alternatively differences) of two random variables, viz. $\bar{\eta}_{k} \equiv \bar{\varepsilon}_{k}+\bar{e}_{k}$, that are mutually independent. In such a case the minimized function will be a weighted sum of quadratic expressions of the form $w_{k}\left\{\left[\varepsilon_{k}-e_{k}(p)\right]+\bar{\eta}_{k}\right\}^{2}$ depending on the probability distributions $\mathcal{D}_{k}\left(\bar{\eta}_{k}\right)$, in the following referred to as 'noise', for the variables $\bar{\eta}_{k}$; the latter are given through the $k$-dependent convolutions

$\mathcal{D}_{k}\left(\bar{\eta}_{k}\right)=\left(P_{k}^{e} * P_{k}^{t}\right)\left(\bar{\eta}_{k}\right)=\int_{-\infty}^{+\infty} P_{k}^{e}\left(\bar{\varepsilon}_{k}-\bar{e}_{k}\right) P_{k}^{t}\left(\bar{e}_{k}\right) d \bar{e}_{k}=\int_{-\infty}^{+\infty} P_{k}^{e}\left(\bar{\varepsilon}_{k}\right) P_{k}^{t}\left(\bar{e}_{k}-\bar{\varepsilon}_{k}\right) d \bar{\varepsilon}_{k}$.

One can see that any further consideration must depend on what we know (or what we agree to accept as reasonable assumptions about) the distributions $P_{k}^{e}\left(\bar{\varepsilon}_{k}\right)$ and $P_{k}^{t}\left(\bar{e}_{k}\right)$. This step is clearly one of the Open Problems that, as we believe, will need to be addressed in detail the future. Here we wish to focus on possibly simplified but effective solutions and thus we reformulate the problem in (7) as follows.

\subsection{Uncertainty distributions and their dynamical transforms}

The idealized, 'true model energies', $e_{n}^{\text {true }}$, although conceptually important, remain in practice unknown elements of the inexact theories, and since finding the 'exact' measurement result is a void issue, we may try to simplify the formulation. Let us replace distributions $\left\{\mathcal{D}_{k}\left(\bar{\eta}_{k}\right)\right\}$ by a new set $\left\{D_{k}\left(\eta_{k}\right)\right\}$ of the new random variables $\eta_{k}$. Doing so we modify the minimisation in (7) by introducing a new function

$$
\chi^{2}(p) \sim \sum_{k} w_{k}\left\{\left[\varepsilon_{k}+\eta_{k}\right]-e_{k}(p)\right\}^{2} .
$$


Let us also introduce a set of samples $\eta_{i} \equiv\left\{\eta_{1}, \eta_{2}, \ldots \eta_{k^{\max }}\right\}_{i}$ for $i=1,2, \ldots N$, each variable sampled according to its $D_{k}\left(\eta_{k}\right)$ distribution. Next by solving (here) the Schrödinger equation we perform $N$ minimisations to find the sets of the optimal parameters $\bar{p}_{i} \equiv\left\{\bar{p}_{1}, \bar{p}_{2}, \ldots \bar{p}_{n_{p}}\right\}_{i}$, the sets of implied eigenvalues $\bar{e}_{k}(i) \equiv\left\{e_{k}\left(\bar{p}_{i}\right)\right\}$ and the corresponding $\chi^{2}\left(\left\{e_{k}\left(\bar{p}_{i}\right)\right\}\right.$. For the ensemble of $\eta_{i}$ samples sufficiently large (below we use $N \sim 20000$ ) we may collect the occurrence histograms such as e.g.

$$
N_{(\bar{\chi}, \delta \chi)}=N\left(\chi \in\left[\bar{\chi}-\frac{1}{2} \delta \chi, \bar{\chi}+\frac{1}{2} \delta \chi\right]\right)
$$

illustrating the quality of the generated spectra in terms of their r.m.s distribution or

$$
N_{\left(\bar{p}_{j}, \delta \bar{p}_{j}\right)}=N\left(\bar{p}_{j} \in\left[\bar{p}_{j}-\frac{1}{2} \delta \bar{p}_{j}, \bar{p}_{j}+\frac{1}{2} \delta \bar{p}_{j}\right]\right)
$$

showing the parametric uncertainties reflecting the ones in the input data and finally

$$
N_{\left[\bar{e}_{k}(i), \delta \bar{e}_{k}(i)\right]}=N\left(\bar{e}_{k} \in\left[\bar{e}_{k}(i)-\frac{1}{2} \delta \bar{e}_{k}(i), \bar{e}_{k}(i)+\frac{1}{2} \delta \bar{e}_{k}(i)\right]\right),
$$

illustrating, in which manner the uncertainties of the individual energy levels are affected. Distributions in Eqs. (10-12) depend on the dynamics of the system through interaction Hamiltonians underlying the equations of motion such as Schrödinger, Dirac, Hartree-Fock etc. For that reason we refer to them as dynamical transforms of the input uncertainty distributions of the inverse problem. In the detailed illustrations we distinguish among the variants such as: the r.m.s. transforms represented by (10), induced parameter-transforms by (11), energy transforms by (12), etc.

It will be instructive to illustrate the above notions using a simple but realistic phenomenological model. We chose for that purpose the neutron single-particle levels in ${ }^{208} \mathrm{~Pb}$ using the phenomenological Woods-Saxon Hamiltonian with parameters given in table 2. The spectrum generated with the latter is then used for the noise testing purposes replacing the true experimental input: we refer to such a spectrum as 'pseudo-experimental'. This offers the advantage of allowing to vary the number of levels to fit in excess of those known experimentally (similar type analysis will need to be performed in the future for other, microscopic theories).

Table 2. Woods-Saxon potential parameters for the neutrons in ${ }^{208} \mathrm{~Pb}$ used to generate the pseudo-experimental levels, among others for the purposes of the noise testing. These parameters reproduce the 'true' experimental levels in Table 1 with the r.m.s. deviation of $0.164 \mathrm{MeV}$ and the maximum error of $0.353 \mathrm{MeV}$.

\begin{tabular}{|c|c|c|c|c|c|}
\hline$V_{o}^{c}$ & $r_{o}^{c}$ & $a_{o}^{c}$ & $\lambda$ & $r_{o}^{s o}$ & $a^{s o}$ \\
\hline-39.520 & 1.371 & 0.694 & 26.133 & 1.255 & 0.500 \\
\hline
\end{tabular}

Let us illustrate the impact of both the number of data points taken for the fit and the broadness of the noise distribution of the data. Illustrations in figure 1 show that if the number of data points is not large enough, the Hamiltonian is capable of adapting itself and of reproducing any noise feature of the experimental input so that the maximum of the distribution corresponds to $\chi^{2}=0$ (error-less fit to 'any' input!). Only when the number of data points is sufficiently large (actually larger than the number of single-particle energies known from today's experiments) can a consistency between the resulting fit distribution and the input noise distribution be (only partially!) achieved. Indeed, observe that (figure 1, right) the 'noisy' input and output distributions lie close, but correspond to the maxima at $\chi \neq 0$. Recall that the noise-less (exact) input generates by definition of the pseudo-experimental spectrum, 

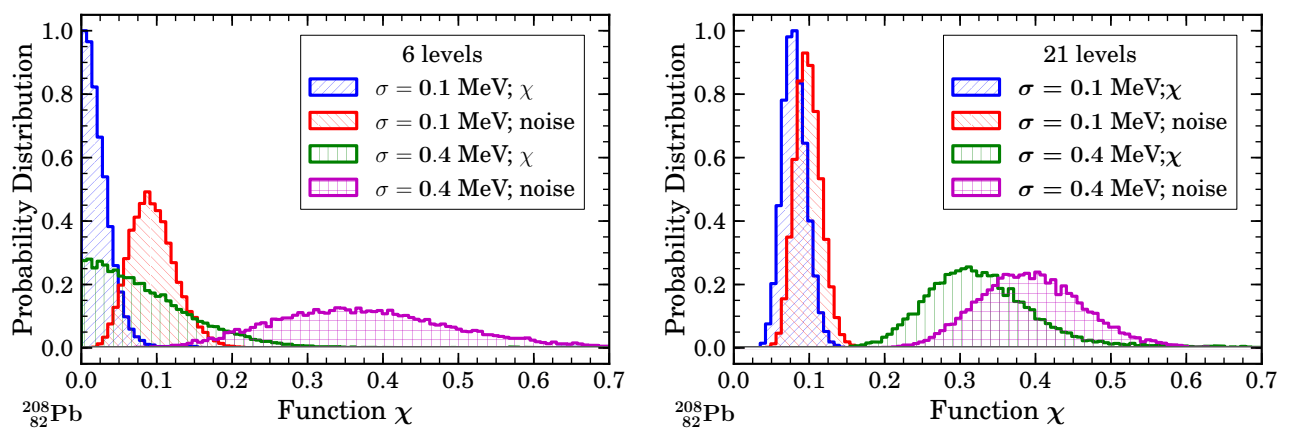

Figure 1. Histograms representing the r.m.s transforms of (10) obtained by fitting to the pseudo-experimental data. In this case we assumed a common 'noise' Gaussian distributions $D_{k}\left(\eta_{k}\right) \rightarrow \mathcal{N}\left(\eta_{k}\right)$, with the width parameters $\sigma=0.1$ and 0.4. Left: the results for 6 levels distributed symmetrically around the Fermi level for the neutrons in ${ }^{208} \mathrm{~Pb}$; Right - similar but using 21 highest-bound levels. The curves are normalized so that the histogram area gives 1 , but scaled, for better legibility so that the highest maximum is at 1 . The label 'noise' refers to the input noise distributions, prior to fitting.

the exact $\chi=0$ solution always. Our observation can be viewed as intuitive: applying a noise with the distribution width $\sigma$ to an exact, pseudo-experimental spectrum, shifts the centroid of the calculated distribution by an increment related to $\sigma$. In all cases an improvement is achieved: the maximum probability is shifted towards smaller $\chi$.

Let us emphasize that the capacity of a Hamiltonian to reproduce a number of energy levels exactly i.e. with $\chi=0$, is not only the question of the number of adjustable parameters $v s$. the number of data points but also the question of adequacy. If the data points form a parabolic dependence in function of a certain parameter while the theory predicts a trigonometric one, even with non-trivially few data points it will not be possible to reproduce the data exactly - unless infinite series are involved.
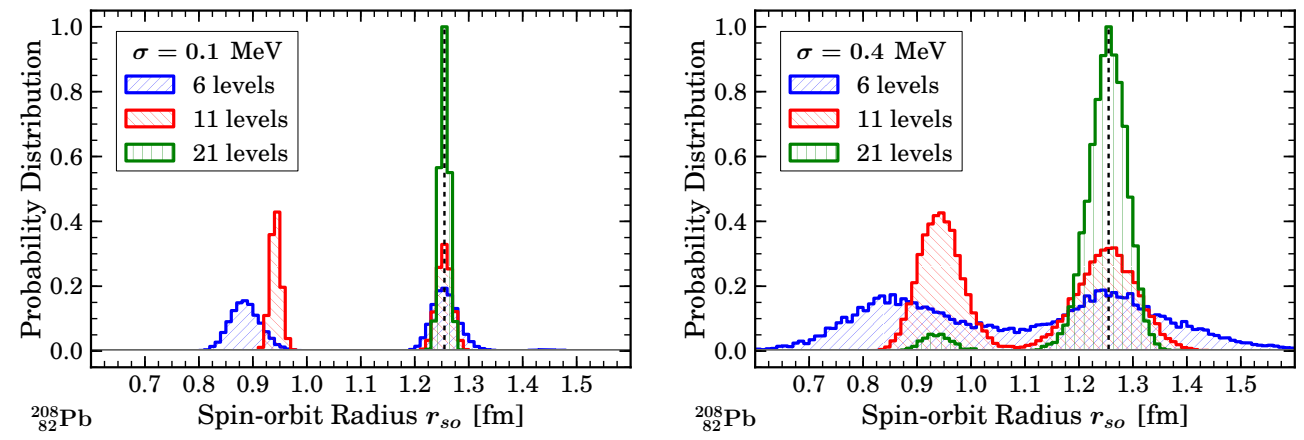

Figure 2. Histograms representing the Schrödinger parameter-transforms of (11) obtained by fitting to 6,11 and 21 pseudo-experimental neutron levels in ${ }^{208} \mathrm{~Pb}$, assuming a common Gaussian noise distribution with $\sigma=0.1 \mathrm{MeV}$, left, and 0.4 $\mathrm{MeV}$, right. Vertical dashed lines give the position of the exact parameter values from table 2. For comments on the double-maximum structure cf. Refs. [11, 12]. 
Examples of the Schrödinger parameter-transforms for a few least-bound neutron levels are shown in figure 2 . Observe the double maximum structure, the so called 'compact' ( $\left.r_{o}^{s o} \approx 0.92 \mathrm{fm}\right)$ and 'normal' or 'non-compact' $\left(r_{o}^{s o} \approx 1.25 \mathrm{fm}\right)$ geometries. This mechanism is interesting and known to the experts: here, not entering into details, it will be sufficient to observe that the single-maximum distribution reflecting the single-maximum distribution of the input noise is obtained only at sufficiently large number of pseudo-experimental levels.
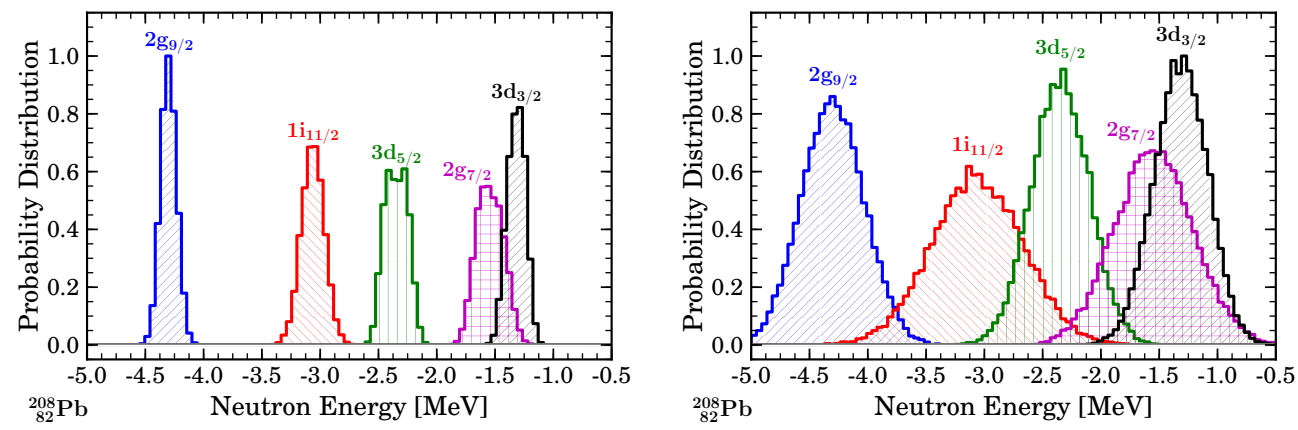

Figure 3. Schrödinger energy-transforms, (12), for a few neutron levels in ${ }^{208} \mathrm{~Pb}$ when performing fits to 11 pseudo-experimental levels with the Gaussian noise distributions of the width $\sigma=0.1 \mathrm{MeV}$ (left) and $0.4 \mathrm{MeV}$ (right).

A few examples of the single-nucleon Schrödinger energy transforms defined through (12) are illustrated in figure 3; observe that the widths corresponding to various levels differ despite the fact that the input uncertainty distribution is common for all the levels in the tests illustrated.

\subsection{Theoretical-level errors caused by experimental-level uncertainties}

It may be (and often is) very useful to know e.g. for the experiment programing, what are the uncertainties in theoretically predicted positions of various levels caused by a possibly large uncertainty in the inverse problem input information about specific levels. For instance, it may happen that a certain experimental error on, say, fitinput $1 i_{11 / 2}$-level, may have no major impact on the results of the fitting algorithm, whereas the same uncertainty on, say $2 g_{7 / 2}$-level, may influence the positions of the fitted levels considerably. Should a similar or any alternative scenario be established, special measurement precision would be required on specific levels and possibly much less so, on some others. This information will usually be very practical to know before establishing the priorities of the related research program - and we give numerous arguments in this article to indicate that the present-day experimental information on the single-nucleon levels in spherical doubly-magic nuclei remains largely an Open Problem for the future.

Information about a 'level-to-level' sensitivity can be obtained through a simple simulation which consists in repeating the adjustment algorithm in function of the displacement of the individual input-level energies (one level at the time). To illustrate the problem we have varied the positions of the input levels of the adjustment algorithm for the neutrons in ${ }^{208} \mathrm{~Pb}$. The illustration of differences between the effects caused by high- $j$ orbitals (here: $1 i_{11 / 2}$ ) and low- $j$ orbitals (here $3 d_{3 / 2}$ ) is presented in 

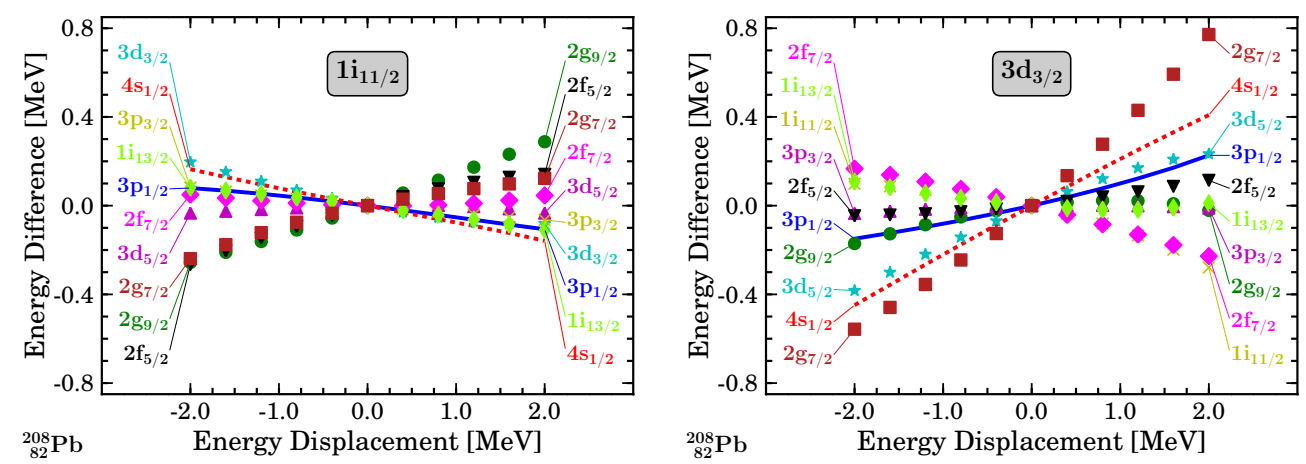

Figure 4. The energy differences as measures of the fit-output uncertainty in the single-particle levels listed - caused by the displacement (as measures of the input-level uncertainty) of the energy of the $1 i_{11 / 2}$-level, left, and the $4 s_{1 / 2}$-level, right. The displacement window of $4 \mathrm{MeV}$ is centred on the original positions of these levels as obtained with the parameters in table 2. Fits using in total 11 pseudo-experimental levels.

figure 4. One may notice that the forms of the over-all dependence are different, e.g. the strongest variation is approximately represented by the interval of only $[-0.3,+0.3]$ $\mathrm{MeV}$ for $1 i_{11 / 2}$ and $[-0.6,+0.6] \mathrm{MeV}$ in the case of the $3 d_{3 / 2}$-levels. Moreover, the strength and the sign of the coupling depend significantly on the quantum numbers of the concerned states: whereas the state $2 f_{5 / 2}$ practically does not couple with $3 d_{3 / 2}$ it is among the strongest-coupling with the level $1 i_{11 / 2}$. It is instructive to compare the

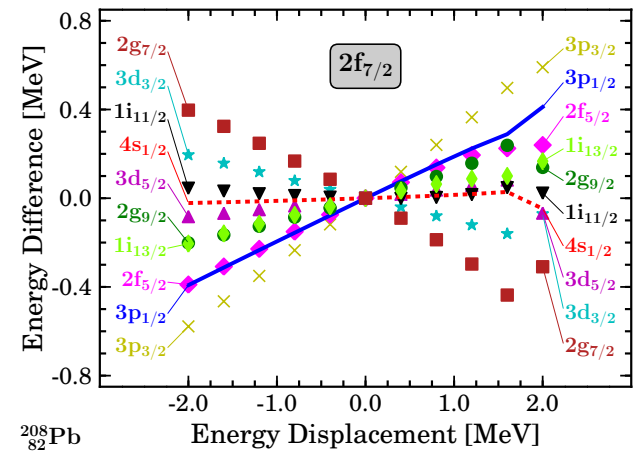

Figure 5. Similar to that in figure 4 but for levels $2 f_{7 / 2}$ (left) and $2 g_{7 / 2}$ (right).

above results with the ones for $2 f_{7 / 2}$ and $2 g_{9 / 2}$ levels, figure 5 . Observe in particular a strong mutual coupling between the $2 f_{7 / 2}$ and $2 g_{7 / 2}$ levels (same sign) and the strong but opposite sign couplings between $3 p_{3 / 2}$ and $2 f_{7 / 2}$, on the one hand, and between $3 p_{3 / 2}$ and $2 g_{7 / 2}$ on the other. The relative parity of orbitals does not seem to have any direct role: observe that the same parity orbitals $1 i_{11 / 2}$ and $1 i_{13 / 2}$ couple with opposite signs to the two considered orbitals in the left- and right-hand side diagrams.

One may conclude that the 'level-to-level' correlations through the adjustment algorithm, if needed e.g. for the predictions and/or extrapolations of the level positions in nuclei away from the tested ones (cf. Sect.4), or for the experiment planning, 
may be very precious but will need to be examined individually, i.e. one level after another. Moreover: these results suggest that the schematic treatment of the noise parametrisation in terms of a common normal distribution is certainly not optimal and will need to be replaced by both experimental and theoretical level-by-level analysis of the uncertainties in order to construct a more intrinsically-consistent procedure.

\section{Predictive power: Examples of neutron spectra in ${ }^{132} \mathrm{Sn}$ nucleus}

The ${ }^{132} \mathrm{Sn}$ nucleus exhibits all the usual characteristics of the doubly-magic nuclei, first of all the high energies of its first excited states. Indeed, the first excited $2^{+}$state lies at $4041 \mathrm{keV}$ and the $3^{-}$state at $4351 \mathrm{keV}$. Moreover, there is only a weak quadrupoleexcitation probability associated with the first $2^{+}$state, with $B(E 2)=7(3)$ W.u. accompanied by the collective octupole-excitation probability with $B(E 3)>7$ W.u.

The four odd-A nuclei close to ${ }^{132} \mathrm{Sn}$ have not been yet studied using one-nucleon transfer reactions. This prevents us to characterize any single-particle content of their states so far identified using $\gamma$-spectroscopic studies following $\beta$-decay, and in some cases by the prompt $\gamma$-decay of nuclei produced as fission fragments.

Considering the lowest excitation energies, denoted $\mathrm{e}_{1}^{e x c}$, as in table 1 , we would like to evaluate the energies $\bar{\varepsilon}$ of all the single-neutron levels around $N_{0}=82$. For that purpose we will use: (i) The conclusions formulated in Sect. 2 for ${ }^{208} \mathrm{~Pb}$, and: (ii) The energy-shifts due to the fragmentations which have been measured, for each level of interest, in the neighbouring isotopes and isotones lying closer to the valley of $\beta$-stability. Single-particle quantities $\bar{\varepsilon}_{\kappa}$ have the similar meaning as the quantities denoted $\varepsilon_{\kappa}$ in the preceding Sections except that in the absence of the adequate experimental information about the neighbours of ${ }^{132} \mathrm{Sn}$, certain information "extrapolated" from the area of ${ }^{208} \mathrm{~Pb}$ will be employed.

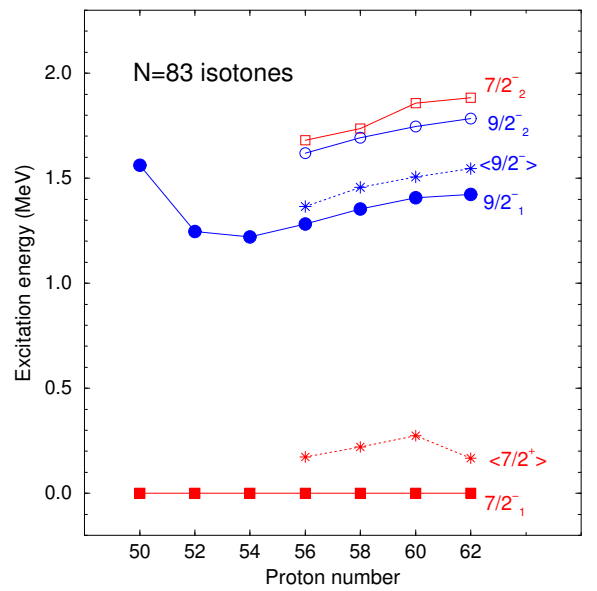

Figure 6. Excited states having $j^{\pi}=7 / 2^{-}$and $9 / 2^{-}$identified in the $N=83$ isotones (from [4]). The average energies (asterisks) are calculated using (2) from the data of $Z=56-62$ nuclei listed in the text.

Let us begin with the example of the two neutron levels lying above the $N_{0}=82$ gap: $\nu f_{7 / 2}$ and $\nu h_{9 / 2}$. In fact two $j^{\pi}=7 / 2^{-}$states and two $j^{\pi}=9 / 2^{-}$states have been measured in the close-lying $N=83$ isotones, ${ }_{56}^{139} \mathrm{Ba}_{83},{ }_{58}^{141} \mathrm{Ce}_{83},{ }_{60}^{143} \mathrm{Nd}_{83}$ and ${ }_{62}^{145} \mathrm{Sm}_{83}$, 
using one-neutron transfer $(d, p)$ reaction on stable targets. The corresponding neutron levels are illustrated in figure 6 , together with the related average energies calculated according to (2): the latter give approximated positions of the expected single-particle neutron-energies. We observe that the fragmentation of the $\nu f_{7 / 2}$ levels results in an energy shift of, approximately, $0.2 \mathrm{MeV}$, whereas the shift associated with the $\nu h_{9 / 2}$ level amounts to, approximately, $0.1 \mathrm{MeV}$.

In a similar fashion many states measured in the $N=81$ isotones and the $Z=51$ isotopes that lie between ${ }^{132} \mathrm{Sn}$ and the $\beta$-stability valley in the $(Z, N)$-plane can be used to obtain the shifts in energy due to the fragmentations of the single-nucleon levels. Some of the results of this type have been illustrated in [13]; all the results for which the data exist today collected in the third column ('shift 1') in table 3.

Table 3. Energies of the first states measured in ${ }_{50}^{133} \mathrm{Sn}_{83}$ and ${ }_{50}^{131} \mathrm{Sn}_{81}$, usually considered as pure single-nucleon states [4]. Excitation energies $\bar{\varepsilon}$ of the singlenucleon states are calculated from the $e_{1}^{e x c}$ energy and the shift in energy due to the fragmentation. The empirical values of the binding energies (B.E.) take into account the values of the total binding energies of the involved nuclei [14]. All the energies are given in $\mathrm{MeV}$. The data written in parentheses are tentative, and since the identification of the corresponding states is rather questionable (new experiments have to be performed to confirm both their energies and their spinparity assignments) these states are ignored in the following discussion.

\begin{tabular}{|c|c|c|c|c|c|}
\hline \multicolumn{6}{|c|}{ Neutron levels around N=82 } \\
\hline Level & \multicolumn{5}{|c|}{ States in ${ }^{133} \mathrm{Sn}$} \\
& $\mathrm{e}_{1}^{\text {exc }}$ & shift $1^{(a)}$ & shift $2^{(b)}$ & $\bar{\varepsilon}$ & B. E. \\
$\nu f_{7 / 2}$ & 0.0000 & 0.2 & $0.6(4)$ & $0.6(4)$ & $-1.9(4)$ \\
$\nu p_{3 / 2}$ & $(0.8537)$ & - & - & - & - \\
$\nu h_{9 / 2}$ & 1.5609 & 0.1 & $0.6(5)$ & $2.2(5)$ & $-0.3(5)$ \\
$\nu p_{1 / 2}$ & $(1.6557)$ & - & - & - & - \\
\hline Level & \multicolumn{5}{|c|}{ States in ${ }^{131} \mathrm{Sn}$} \\
\hline & $\mathrm{e}_{1}^{\text {exc }}$ & shift $1^{(a)}$ & shift $2^{(b)}$ & $\bar{\varepsilon}$ & $\mathrm{B} . \mathrm{E}$. \\
$\nu d_{3 / 2}$ & 0.0000 & 0.25 & $0.6(4)$ & $0.6(4)$ & $-7.9(4)$ \\
$\nu h_{11 / 2}$ & $0.0651^{(c)}$ & 0.3 & $0.6(3)$ & $0.7(3)$ & $-8.0(3)$ \\
$\nu s_{1 / 2}$ & 0.3317 & 0.25 & $0.6(4)$ & $0.9(4)$ & $-8.2(4)$ \\
$\nu d_{5 / 2}$ & 1.6545 & - & $0.6(4)$ & $2.3(4)$ & $-9.6(4)$ \\
$\nu g_{7 / 2}$ & 2.4341 & - & $0.6(4)$ & $3.0(4)$ & $-10.3(4)$ \\
\hline
\end{tabular}

${ }^{(a)}$ Shifts in energy due to the fragmentation measured in neighbouring nuclei. ${ }^{(b)}$ The values obtained through analogy by extrapolating from the data on ${ }^{208} \mathrm{~Pb}$. The numbers in parentheses give errors in the last digit. ${ }^{(c)}$ From [15] .

Another possibility of the order of magnitude estimates of the energy-shifts is to use the same proportions of the $2^{+}$and $3^{-}$excitation energies as the ones found in the ${ }^{208} \mathrm{~Pb}$ nucleus (cf. Sect. 2). Using these proportions we obtain the shifts of the order of $0.6 \mathrm{MeV}$ ( $15 \%$ of $4 \mathrm{MeV}$ ), for both the single-particle states coupled through $\Delta l=2$ (and $\Delta j=2$ ) and those coupled through $\Delta l=3$ (and $\Delta j=3$ ). These estimates are given in the fourth columns ('shift 2') in table 3, together with the estimated errors. The errors have been determined in such a way that the values of 'shift 1' and 'shift 2' 
are consistent within the error bars. Finally the empirical values of binding energies (B.E.) of the nucleon levels in the potential well of ${ }^{132} \mathrm{Sn}$ are calculated using the total binding energies of the involved nuclei $[14]$ and the $\bar{\varepsilon}$ values.

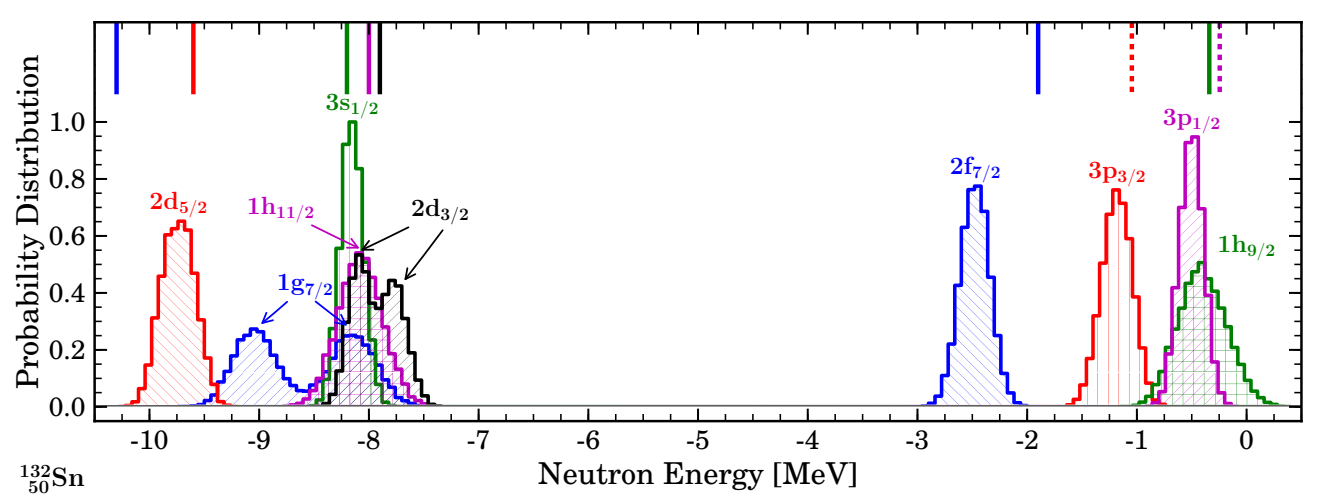

Figure 7. Results of the extrapolation from the ${ }^{208} \mathrm{~Pb}$ to the ${ }^{132} \mathrm{Sn}$ nucleus for the neutrons. With a sample of $N=20000$ sets $\eta_{i}$, cf. the comments around equations (10-12), the parametric histograms resembling those in figure 2 have been obtained (using 11 level simulation); the noise width $\sigma=0.1 \mathrm{MeV}$. With each of the so obtained $N=20000$ sets of parameters the results for the neutrons in ${ }^{132} \mathrm{Sn}$ nucleus have been obtained. Solid bars give positions of experimetal levels from table 3 , dashed bars give positions of experimentally uncertain levels.

We have performed the calculations of the neutron levels in ${ }^{132} \mathrm{Sn}$ using the parameters of the 'well studied' nucleus ${ }^{208} \mathrm{~Pb}$ together with the uncertainties of the theoretical predictions represented in the form of the histograms that resemble the ones presented earlier. The results are illustrated in figure 7 . Let us observe that except for the orbitals $1 g_{7 / 2}$ and $2 f_{7 / 2}$, the ones that were discussed in the preceding Section with the conclusion that they respond rather sensitively to the input errors all other levels lie close to the experimental positions. Moreover, the newly estimated positions of the levels from table 3 (marked with the dashed lines) lie closer to the theory predictions. Another conclusion at this step of the analysis is that the initial input uncertainties with the width $\sigma$ of $0.1 \mathrm{MeV}$ was most likely slightly too optimistic: a value of the order of $\sigma \sim 0.15$-to- $0.2 \mathrm{MeV}$ corresponds better to the estimated uncertainties.

Figure 7 can be seen as a prototype of the future illustrations of theory results that include the cumulative theory and experiment uncertainties of the modelling. Such diagrams, completed by the experimental uncertainty distributions that would replace the dashed- and full-bars in the upper part of the figure would offer a much more satisfying representation of our ignorance. The corresponding information (let us emphasize: subjective, as the very notion of probability) would be nevertheless the precious starting element for the planing of improvements. The levels identified in this way as poorly known, with too broad uncertainty distributions, should be re-examined with priority. Similarly, the levels manifesting an 'unusual' structure of their histograms like the one for the $1 g_{7 / 2}$ level in figure 7 , should be re-examined from the point of view of the quantum origin by, first of all, identifying the responsible interaction: central, spin-orbit, tensor etc. Studies of the spectral properties of the nuclear Hamiltonians and their spectroscopic predictive power, as illustrated above, represent a series of, in our opinion fascinating, Open Problems for the near future. 


\section{Instabilities of adjustment algorithms and ill-posed inverse problems}

The problem of stability of the parameter optimisation depends not only on the form of the Hamiltonian but also on the number and the characteristics of the data points. In applied mathematics this problem is related to the notion of ill-posedeness of the inverse problem and it will be instructive to present its basic elements briefly.

Consider a normalised $\chi^{2}$-function obtained from the one in (9) by removing the error (noise) factor $\eta$ and fixing the normalisation in accordance with the literature, cf. e.g. $[16]$ :

$$
\chi^{2}(p)=\frac{1}{\left(k_{\varepsilon}-n_{p}\right)} \cdot \sum_{k=1}^{k_{\varepsilon}} w_{k}\left[\varepsilon_{k}-e_{k}(p)\right]^{2} ;
$$

as before, $k_{\varepsilon}$ is the number of experimental levels and $n_{p}$ the number of parameters. Assuming that we are not too far from the solution we can linearize the problem

$$
e_{k}(p) \approx e_{k}\left(p_{0}\right)+\left.\sum_{j=1}^{n_{p}}\left(\frac{\partial e_{k}}{\partial p_{j}}\right)\right|_{p=p_{0}}\left(p_{j}-p_{0, j}\right)
$$

according to the standards of the Newton algorithms. Abbreviating the notation

$$
\left.J_{k j} \equiv \sqrt{w_{k}}\left(\frac{\partial e_{k}}{\partial p_{j}}\right)\right|_{p=p_{0}} \text { and } b_{k}=\sqrt{w_{k}}\left[\varepsilon_{k}-e_{k}\left(p_{0}\right)\right],
$$

we may transform (13) into the form

$$
\chi^{2}(p)=\frac{1}{\left(k_{\varepsilon}-n_{p}\right)} \cdot \sum_{k=1}^{k_{\varepsilon}}\left[\sum_{j=1}^{n_{p}} J_{k j} \cdot\left(p_{j}-p_{0, j}\right)-b_{k}\right]^{2} .
$$

It is straightforward to show that the necessary conditions for the minimum of $\chi^{2}$ are equivalent to the matrix relation (cf. e.g. [17]):

$$
\frac{\partial \chi^{2}}{\partial p_{i}}=0 \rightarrow\left(J^{T} J\right) \cdot\left(p-p_{0}\right)=J^{T} b \text { with }\left[J_{k j}\right] \equiv J
$$

Thus the inverse problem takes the algebraic form with the schematic structure

$$
J \cdot\left(p-p_{0}\right)=b \leftrightarrow p-p_{0}=J^{-1} b .
$$

Recall that $J$ is in general rectangular $k_{\varepsilon} \times n_{p}$ matrix whereas $p \in \mathbb{R}^{n_{p}}$ and $b \in \mathbb{R}^{k_{\varepsilon}}$.

To illustrate the problem of ill-conditioning we will remind the reader of the socalled Singular Value Decomposition. Recall that any rectangular matrix $A$ can be decomposed as a product of three matrices (cf. e.g. [1, 2] and references therein)

$$
A=U \cdot D \cdot V^{T} \text { with } U \in \mathbb{R}^{m \times m}, \quad V \in \mathbb{R}^{n \times n}, \quad D \in \mathbb{R}^{m \times n},
$$

where the diagonal matrix $D$ can be represented with the help of the eigenvalues $\left\{d_{j} ; j=1,2, \ldots \min (m, n)\right\}$ and written down symbolically in the form:

$$
D=\operatorname{diag}\left\{d_{1}, d_{2}, \ldots d_{\min (m, n)}\right\} .
$$

Applying the above decomposition to a real, orthogonal matrix $J$ for which $J^{-1}=J^{T}$ :

$$
\left(p-p_{0}\right)=J^{T} b \leftrightarrow J^{T}=V \cdot D^{T} \cdot U^{T}
$$

ๆ Ill-posedeness of the inverse problem leads to instability of the parameter adjustment procedures: narrow distributions of the input noise leading to broad distributions in terms of outcome parameters. The instability manifests itself also through the divergence of the parameter correlation matrix and that of confidence intervals - obviously highly undesired effects. 
where

$$
D^{T}=\operatorname{diag}\left\{\frac{1}{d_{1}}, \frac{1}{d_{2}}, \ldots \frac{1}{d_{p}} ; 0,0, \ldots 0\right\} .
$$

In other words, if the diagonal matrix $D$ in the decomposition of $J$ has very small or zero eigenvalues, actually quite a common case, then $J^{-1}$ in (18) is getting singular and no stable solution for the Hamiltonian parameters can be obtained. We say that the inverse problem is ill-posed.

It follows from linear algebra that the above singularity manifests a correlation: one or more components of $p$ are functions of the others. The singularities and thus the possible destabilizing inter-dependence among the parameters depend in turn on the number and the characteristics of the data points, and as we will point out shortly, even one parameter-divergence of this type causes the divergence of the whole correlation matrix. Indeed, it can be shown [16] that the correlation matrix takes the form

$$
\left\langle\left(p_{i}-\left\langle p_{i}\right\rangle\right) \cdot\left(p_{j}-\left\langle p_{j}\right\rangle\right)\right\rangle \sim \chi^{2}(p)\left(J^{T} J\right)_{i j}^{-1},
$$

whereas from Eqs. (20)-(22) it is possible to show that

$$
\left[\left(J^{T} J\right)^{-1}\right]_{i j}=\sum_{\ell=1}^{r} V_{i \ell} \frac{1}{d_{\ell}^{2}}\left[V^{T}\right]_{\ell j} .
$$

If in $(22)$ one or more $d_{k} \rightarrow 0$, then $\left(J^{T} J\right)^{-1}$ tends to infinity and generally, the confidence intervals $\left(p_{i}-\left\langle p_{i}\right\rangle\right)$ of all parameters diverge. This conclusion shows once again the importance of analysis of the statistical significance of the parameter adjustment procedures if the predictive power of the resulting Hamiltonian is aimed at. We believe that this is one of the important Open Problems in today's progress in the physically meaningful applications of the nuclear microscopic theories.
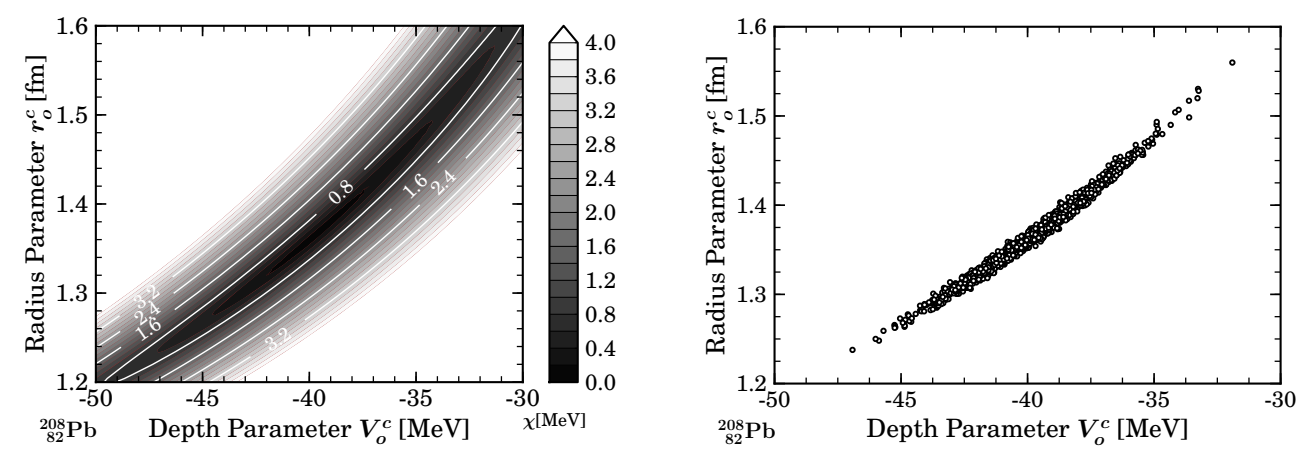

Figure 8. Left: Illustration of the $\chi^{2}$-test in function of the depth and radius parameters of the central Woods-Saxon potential for the neutrons in ${ }^{208} \mathrm{~Pb}$, evaluated for 11 levels close to the Fermi energy. The remaining four parameters of the potential are fixed at the values from table 2. Right: Results of the $\mathrm{N}=20000$ minimisations over all the six parameters of the potential to fit the same 11 pseudo-experimental levels as in the left-hand side, each time changing the noise hypothesis (cf. discussion around Eqs. (10-12)). Observe the same parametric correlations visible from both types of tests.

It can easily be shown that the presence of inter-parametric correlations manifest itself in the form of deep valleys of the $\chi^{2}$-test plots that can be illustrated in terms of projections onto certain hyper-planes in the space of $p \in \mathbb{R}^{n_{p}}$. Illustration of this type is shown in figure 8 (left) for the correlation between the radius and the potential 
depth parameters of the central potential in the case of the Woods-Saxon Hamiltonian with all other remaining parameters fixed. The generalisation of this test for the case of minimisation over all adjustable parameters does not change the conclusion: the two parameters are strongly, but not fully correlated; indeed, the full, exact correlation would imply that the bottom of the valley rest at the zero-value. Let us emphasize that the details of the behaviour change with the number of the experimental levels taken into considerations.

We may remark at this point that: a. Examining of the possible presence of the parametric correlations is a prerequisite; only after having established the stability of the adjustment, cf. Eqs. (22-23), we may decide about the next steps of the procedure; b. Fortunately, the methods of regularization of the unstable algorithms are copiously described in the textbooks and specialized literature - it is usually sufficient to select the optimal approach. But the solution of the problem begins with the first item above rather than proceeding according to the 'chi-by-the-eye' approach mentioned in the third footnote of this article.

\section{Summary - Predictive power of nuclear Hamiltonians: Open Problems}

We have discussed the question of the statistically-significant determination of the nuclear physics Hamiltonians through conscious adjustment of its free parameters the method known in applied mathematics as the inverse problem. In contrast to sometimes formulated opinions, these are the inherent theory and modelling errors that lead to the most important parametric uncertainties of the Hamiltonians and not necessarily the experimental errors. In our analysis we have distinguished between the general discussion of the impact of theoretical and experimental errors on the theory's predictive power and a specific realm of applications: Illustrations using the example of properties of single-nucleon levels of doubly-magic spherical nuclei.

The article advocates the new way of formulating and interpreting the theory predictions: not only provide the theory's numerical results (the present day situation) but provide also their statistical significance represented e.g. by probability(ies) with which the theoretical predictions are expected to apply in nature.

The $1^{\text {st }}$ series of Open Problems consists then in constructing the error probability distributions that would quantify in the best possible, even though always a subjective way, the degree of the missing knowledge. In the application to the single-nucleon spectra, it would be important to extend the analysis to all doubly-magic spherical nuclei, gradually and systematically including the impact of pairing correlations, coupling with the surface oscillations (quadrupole and octupole), as well as the coupling with the continuum for the weakly bound states. The generalized modelling would include both the Hartree-Fock and phenomenological mean field theories as the first step.

For a given Hamiltonian, the functioning of the parameter adjustment algorithms depends both on the number of data points as well as on their intrinsic characteristics (such as e.g. deeply bound nucleonic states, high- $j$ or low- $j$ orbitals etc.) vs. the number of parameters. Hamiltonians that can be called adequate in the context are able to exactly reproduce the non-trivial number of data points, for instance typically 6-to-8 single-nucleon levels in all the doubly magic nuclei when using Woods-Saxon potentials. We have demonstrated that for these low numbers of data points, the discussed Hamiltonians are also capable of reproducing an infinite number of sets of data points obtained from a selected initial set by a random noise perturbation. 
Such Hamiltonians reproduce then (within relatively broad limits) an arbitrary noise exactly. It is therefore necessary to establish the lower limit for the numbers of levels in the fit-input data set so that the Hamiltonian can be really constrained. On the basis of the Woods-Saxon Hamiltonian tests we could establish that this number exceeds the number of experimentally known single-particle levels even in the relatively well studied ${ }^{208} \mathrm{~Pb}$ nucleus.

The $2^{\text {nd }}$ series of Open Problems, specifically addressing the single-nucleon subfield of the project, would consist in as extended as possible an experimental study of the new average one-nucleon energies introduced in section 2. Alternatively, a similar type of information could be obtained from studying the so-called band-head energies in the deformed nuclei; those also constrain specifically the mean-field Hamiltonians and combining the two approaches seems a valuable complementary counter-checking research to perform.

The $3^{\text {rd }}$ series of Open Problems concerns an important general aspect of the proposed new strategy and techniques: examining the inter-dependences among some parameters of the Hamiltonian that remain 'hidden' i.e. we can discover them only after having performed an analysis of the type discussed in section 5. This mechanism, as discussed in the article, depends not only on the structure of the Hamiltonian but also on the samples of the data used to solve the inverse problem - and thus should be discussed on the case-by-case basis. Its importance consists in the fact that in the presence of inter-relations, the parametric confidence intervals diverge rendering the obtained Hamiltonians unsuited for extrapolations into the new areas of nuclei.

Acknowledgements. Support within the collaboration project TetraNuc through the $\mathrm{IN}_{2} \mathrm{P}_{3}$, France and through exchange program between $\mathrm{IN}_{2} \mathrm{P}_{3}$ and COPIN, Poland, is acknowledged.

\section{References}

[1] Calvetti D and Somersalo E 2007 Introduction to Bayesian Scientific Computing (Surveys and Tutorials in the Applied Mathematical Sciences Vol. 2) (New York: Springer Science+Business Media)

[2] Press W H, Teukolsky S A, Vetterling W T and Flannery B P 1992 Numerical Recipes in FORTRAN 77: The Art of Scientific Computing (Cambridge: Cambridge University Press)

[3] Baranger M 1970 Nucl. Phys. A 149225

[4] ENSDF data base, http://www.nndc.bnl.gov/ensdf/

[5] Galès S et al. 1985 Phys. Rev. C 3194

[6] Langevin-Joliot H et al. 1993 Phys. Rev. C 471571

[7] Galès S et al. 1978 Phys. Rev. C $\mathbf{1 8} 2475$

[8] Tickle R and Gray W S 1975 Nucl. Phys. A 247187

[9] Grabmayr P et al. 1992 J. Phys. G: Nucl. Part. Phys. 181753

[10] Hamamoto I 1974 Phys. Rep. C 1063

[11] Dudek J, Rybak K, Szpak B, Porquet M-G, Molique H and Fornal B 2010 Int. J. of Modern Physics $\mathrm{E}$ in print

[12] Szpak B, Dudek J, Porquet M-G, Rybak K, Molique H and Fornal B 2010 Int. J. of Modern Physics E in print

[13] Sorlin O and Porquet M-G 2008 Prog. Part. Nucl. Phys. 61602

[14] Audi G, Wapstra A H and Thibault C 2003 Nucl. Phys. A 729337

[15] Fogelberg B et al. 2004 Phys. Rev. C 70034312

[16] Björck A 1996 Numerical Methods for Least-Squares Problems (Philadelphia: Society for Industrial and Applied Mathematics)

[17] Vogel C R 2002 Computational Methods for Inverse Problems (Philadelphia: Society for Industrial and Applied Mathematics) 